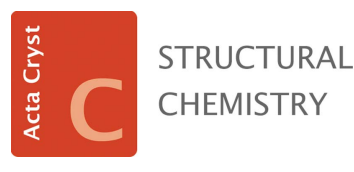

ISSN 2053-2296

\title{
Improving the solubility of berberine
}

\author{
José A. Fernandes*
}

Quinta do Anjo, Portugal. *Correspondence e-mail: jafernandes1974@gmail.com

Keywords: berberine; solubility; API; cocrystal

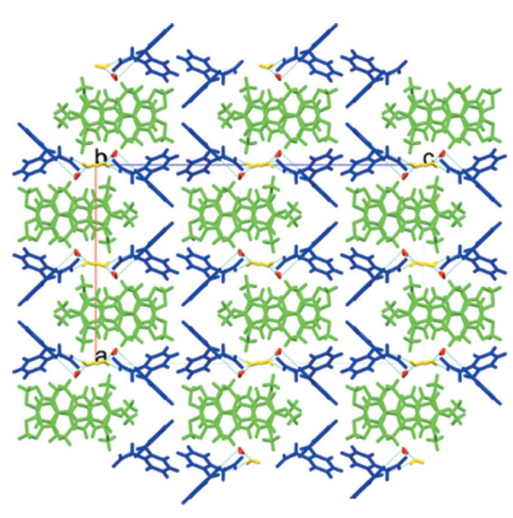

(C) 2020 International Union of Crystallography
While a certain compound can be considered as an active pharmaceutical ingredient (API) in controlled situations, it may fail as a medicine due to problems related to its physical, chemical or biological properties. Moisture, heat or oxygen may jeopardize the chemical or physical stability of the compound, compromising its long-term storage. On the other hand, the form in which the compound is available may present poor solubility in water, preventing its absorption by the human body. The preparation of cocrystals is among the solutions that have been used to circumvent these kinds of problems. If one of the components of a cocrystal is an API, the crystal is classified as a pharmaceutical cocrystal. Conversely, the other component is called a coformer. It is the screening of the vast number of available coformers that allows the optimization of the target parameter (e.g. improving stability toward moisture).

In spite of berberine (BBR) being a water-soluble drug with a wide spectrum of medical applications, it presents poor intestine absorption via oral administration (Fratter \& De Servi, 2014). Additionally, this API is highly hygroscopic and presents an extremely bitter taste. Cocrystals have been prepared in attempts to solve these problems ( $\mathrm{Lu}$ et al., 2019; Wang et al., 2016).

Recently, in Acta Crystallographica Section C, Sun et al. (2019) proposed a possible solution for improving the water solubility of BBR by the formation of multicomponent pharmaceutical salts (or ionic cocrystals in a wider definition; Kumar \& Nanda, 2018) with diclofenac (DIC), another known API, as coformer. Thus, they managed to prepare DIC salts of BBR as a dihydrate (denoted I) and four other solvates [hemidichloromethane dihydrate (II), ethanol (III) and methanol (IV and V, mono- and disolvates, respectively)]. These salts were readily prepared by slow evaporation of mixtures containing the corresponding solvents.

All crystals show an $\mathrm{N}-\mathrm{H} \cdots \mathrm{O}$ hydrogen-bond interaction between the amino group of DIC and the carboxylate group of BBR comprising an $S(7)$ graph-set motif. Additionally, $\mathrm{O}-\mathrm{H} \cdots \mathrm{O}$ hydrogen-bond interactions are also present in I, II and V, comprising rings with $R_{4}^{4}(12)$ and $R_{6}^{4}(12)$ graph-set motifs in I, a one-dimensional chain in II and an isolated hydrogen bond in V. Additionally, $\pi-\pi$ interactions are also present in all the compounds, except for II.

Sun et al. (2019) have contributed to the library extension of berberine presentations. Further studies may result in the use of the prepared compounds for new formulations of berberine with improved properties.

\section{References}

Fratter, A. \& De Servi, B. (2014). Int. J. Drug Deliv. Technol. 5, 33-42.

Kumar, S. \& Nanda, A. (2018). Mol Cryst. Liq. Cryst. 667, 54-77.

Lu, Q., Dun, J., Chen, J., Liu, S. \& Sun, C. C. (2019). Int. J. Pharm. 554, 14-20.

Sun, W., Zuo, L., Zhao, T., Zhu, Z. \& Shan, G. (2019). Acta Cryst. C75, 1644-1651.

Wang, C., Perumalla, S. R., Lu, R., Fang, J. \& Sun, C. C. (2016). Cryst. Growth Des. 16, 933-939. 